\title{
Frontiers General Neurology Grand Challenge - a generalist in a specialized world
}

\author{
Murray Flaster* \\ University Medical Center of Southern Nevada, University of Nevada School of Medicine, Las Vegas, NV, USA \\ *Correspondence: murray.flaster@umcsn.com
}

Neurologists, like all physicians live in a world of increasing specialization and subspecialization. This is a response to progress, the result of our increased understanding of pathophysiology and the expansion of diagnostics, therapeutics, and interventions. For the patient, subspecialization offers the promise of a very precise answer to a specific clinical complaint or condition but simultaneously poses the problem of reiterative visits to multiple subspecialists and the integration of findings into a single plan of patient care. For the individual neurologist, it poses the question of which subspecialty skills to combine, and when to refer or defer. Furthermore, we recognize that every subspecialist is a generalist when confronted with a neurological problem anywhere outside one's particular expertise.

Some current sources divide Neurology into as many as 32 subspecialties. Frontiers thus far recognizes 16 neurologic subspecialties by disease grouping, for example epilepsy or movement disorders, and 5 subspecialties by approach or venue, for example interventional neurology, teleneurology, or hospital neurology. The American Academy of Neurology recognizes 25 subsections at last count.

Within that mosaic of knowledge and practice pattern, how should we best define the current scope and role of General Neurology? A listing for a fellowship in General Neurology at a preeminent university Department of Neurology notes that it is needed for basic diagnosis and triage within major centers. Another source notes the inevitability of General Neurology in smaller communities where subspecialties per force are underrepresented. On the other hand, the American Academy of Neurology General Neurology Section states that general neurology "represents the Neurologist in its purest form". This would imply that a broader "general" perspective imparts an advantage both to the patient and to the practitioner.

The grand challenge that the Frontiers General Neurology editors envision is providing a platform for both opinion and rigorous investigation of the following issues:

Is there a best or preferred skill set for the general neurologist? What scope of practice yields best results for patients, patient endorsement ("satisfaction"), best system efficiency or best business model.

For each topical area in neurology be it dementia, epilepsy, movement disorders, neuroimmunolgy, neuromuscular disease, cerebrovascular disease or other subspecialty area, can we define a General Neurology fund of knowledge and scope of practice? Can we define best practices for subspecialty referral? Can we measure optimal care (Vickrey et al., 1999; Tan et al., 2010)? It is assumed that specialty care offers advantages. Can these advantages be measured? Can simple modifications or additions to Generalist management significantly reduce these differences if they are shown to exist?

What potential roles are there for clinic restructuring, informatics, or perhaps the adoption of a "medical home" (American College of Physicians: http:// www.acponline.org/advocacy/?hp) for chronic neurologic conditions in the quest for improved general neurological care? Can virtual clinics be created that combine local general and remote specialty neurologic care by the creative use of telemedicine and informatics? Such a virtual clinic may optimize quality and efficiency of care while promoting patient comfort and trust and preserving professional familiarity with patient, family, and local circumstances.

Frontiers General Neurology welcomes contributions in these areas including scope of care and best practices originated by either experts or generalists. Of course, as progress is made, we would hope that reviews, proposals, and opinions would be reinforced by results of careful and creative investigation.

\section{REFERENCES}

Tan, H., Yu, J., Tabby, D., Devries, A., and Singer, J. (2010). Clinical and economic impact of a specialty care management program among patients with multiple sclerosis: a cohort study. Mult. Scler. 16, 956-963.

Vickrey, B. G., Edmonds, Z. V., Shatin, D., Shapiro, M. F., Delrahim, S., Belin, T. R., Elison, G. W., and Myers, L. W. (1999). General neurologist and subspecialist care for multiple sclerosis, patients' perceptions. Neurology 53, 1190-1197.

Received: 24 August 2010; accepted: 16 September 2010; published online: 25 October 2010.

Citation: Flaster M (2010) Frontiers General Neurology Grand Challenge-ageneralist in a specialized world. Front. Neur. 1:134. doi: 10.3389/fneur.2010.00134

This article was submitted to Frontiers in General Neurology, a specialty of Frontiers in Neurology.

Copyright (c) 2010 Flaster. This is an open-access article subject to an exclusive license agreement between the authors and the Frontiers Research Foundation, which permits unrestricted use, distribution, and reproduction in any medium, provided the original authors and source are credited. 